Oliviero. Über ein Verfahren zur Kultur des. Gonokokkus. Gazette médicale de Paris. 1913. Nr. 194.

Verfasser versetzt $10 \mathrm{ccm}$ des zu untersuchenden Urins mit $1 \mathrm{ccm}$ Pferdeserum; nach 12 Stunden im Brutschrank $\left(36-37^{\circ}\right)$ zeige sich ein wolkiges Depot, nach 18 Stunden könne eine mikroskopische Untersuchung vorgenommen werden. Oliviero vermutet, daß der Gonokokkus die Fähigkeit hat, in der Urethra Saprophyt zu werden und gemeinsam mit dem Micrococcus ureae und Bazillus ureae eine ammoniakalische Gärung des Urins zu veranlassen.

Theodor Schwab (Karlsruhe).

\title{
Nicht venerische Erkrankungen der Harn- und Geschlechtsorgane.
}

Boulanger, L. Über die Diagnostik und Therapie von Schleimhautfissuren der Urethrabei chronjscher as eptischer Urethritis. Gazette médicale de Paris. 1913. Nr. 192.

Als Symptome bezeichnet Verfasser Sekretion der Harnröhre morgens, mehr weniger Fäden in der ersten Urinportion morgens, bei Sondierung und Urethroskopie Blatungen verschiedener Stärke. Boulanger behandelt die kranken Stellen im Urethroskop mit Lapis in Substanz in 8tägigen Intervallen bis. zur Vernarbung; nachher ist eine Bougieskur wegen der Striktur anzuschließen. Patient ist auf die lange Dauer des Leidens. und damit auch der Behandlung aufmerksam zu machen.

Theodor Schwab (Karlsruhe).

Delcroix de Coster. Über einen Fall von plastischer Induration der Corpora cavernosa. Gazette médicale de Paris. 1913. Nr. 194.

Interessant ist an dem Falle, daß, während Heißluft, heiße Kompressen eine erhebliche Besserung nicht brachten, unter einer Tuberkulinbehandlung innerhalb $3 \frac{1}{2}$ Monaten die Indurationen ganz verschwanden. Pat. war hereditär belastet, Vater und Bruder an Tuberkulose gestorben.

Theodor S chwab (Karlsxuhe).

André, P. und Boeckel, André. Über die Heilung eines Falles vonHarnröhrenfistel durch zeitweise Ableitung des Urins am Perineum und Urethroplastik in einer Sitzung. Gazette médicale de Paris 1913. Nr. 198. Kasuistik.

Theodor $\mathrm{Sch}$ wab (Karlsruhe).

Courtade. Ein neuer Béniqué zur Ausführung der elektrolytischen Dilatation der Urethra. Journ. d. méd. de Paris. 1913. Nr. 10. 
Mit den gewöhnlichen Metallknopfsonden zur zirkulären Elektrolyse kommt man bisweilen nicht bis zur Stelle der Verengerung, weil bei Anwendung der dünnen Kaliber oder bei geschlängeltem Verlauf der Harnröhre leicht Spasmus der Urethra membracea eintritt. Wo dies der Fall, hilft Armierung der Bougies mit einer Leitsonde über die Schwierigkeiten hinweg. Da aber die Befestigung mittels eines Schraubengewindes nicht ungefährlich sein würde, hat $\mathrm{Courtade}$ besondere Béniqués aus Seide mit einem durchgehenden Kanal von Eynard als Leitsonden konstruieren lassen.

F. Münchbeimer (Wiesbaden).

Hainer. Ein seltener Frem dkörper in der männlichen Harnröhre. Münch. med. Wochenschr. 19l3. Nr. 10.

Ein 70jähriger Mann führte sich in die Urethra eine $18 \mathrm{~cm}$ lange, $7 \mathrm{~mm}$ dicke Hutnadel ein, die durch Urethrotomia externa entfernt wurde. Theodor B a er (Frankfurt a. M.).

Ceni, A. Spermatogenetische Aberration infolge traumatischer Gehirnerschütterung. VIII. Vereinigung der ital. Gesellschaft für Pathologie. Pisa 25--28. März 1913.

Infolge traumatischer Gehimerschütterung hört nach Ceni beim Hunde sehr häufig der physiologische spermatogenetische Proze $B$ auf und es beginnt ein abnormer spermatogenetischer Prozeß.

Die Samentochterzellen und besonders die Spermatozyten in Prophase und Metaphase, erleiden eine ganz besondere funktionelle Aberration, anstatt in ihrer komplizierten Evolution, die durch die Spermatiden hindurch zu den Spermatozoen führt, fortzufahren. Die Chromosomen dieser Zellen gehen aus dem filamentären Zustande bald in den emes kleinen rundlicben Körperchens über, das durch verschiedene Phasen hindurch sich in eine abgeplattete chromatische, mit einem zentralen ensinophilen Hof ausgestattete Scheibe verwandelt.

Während dieser Transformation des zellulären Idioplasmas erfährt der Zellkörper, der bedeutend an Volumen zunimmt, einen AszensionsprozeB, indem er sich in die oberen Schichten des Epithels der Samenkanälchen verlegt, bis er in das Lumen der Kanälchen eintritt. In dieser Phase erleidet der Zellkörper eine evidente Disgregation, und oft werden die oben erwähnten scheibenförmigen chromatischen Körperchen in Freiheit gesetzt und als solche durch die Ducti efferentes, Epididymis etc. eliminiert. Am häufigsten jedoch wird die aberrierende Samenzelle fast intakt eliminiert, bevor die aberrierenden Spermatozoen in hreiheit gesetzt sind.

Nicht selten endlich erfahren diese aberrierenden Spermatozoen vor ihrer Elimination einen ganz besonderen Involu- 
tionsprozeß. Die periphere chromatische Scheibe, welche die histochemischen Reaktionen des nurmalen Spermatozoenkopfes zeigt, verliert allmählich diese Eigenschaften und verdiunnt sich, bis sie, wenn auch nicht vollständig, so doch zum großen Teile verschwindet, während ihr zentraler eosinophiler Hof sich in ein lichtbrechendes Körperchen transformiert, das sich sowohl mit den basischen, als auch mit den saueren Substanzen, aber besonders mit den letzteren färbt.

J. Ullmann (Rom).

Todde, C. Die männlichen Geschlechtsdrüsen bei den Geisteskrankheiten. VIII. Vereinig. der ital. Gesellschaft für Pathologie. Pisa 25-28. März 1913.

Die makro- und mikroskopischen Untersuchungen Toddes waren auf das Studium der männlichen Geschlechtsdrüsen von 200 mit verschiedenen Formen von Geisteskrankheiten behafteten Individuen gerichtet und bezogen sich auf folgende Gruppen: Paralysis progr. 28, Psychosis pellagrosa 12, P. alcobolica 30, P. epileptica, Dementia senilis 84, maniakalischdepressives Irresein 20, Dementia praecox 25 , sekundäre oder konsekutive Demenz 12, Idiotie oder Imbezillität 20.

$\mathrm{Zu}$ Kontrolluntersuchungen bediente sich A. der Hoden von gesunden, zufällig verstorbenen (5 Fälle) und von an gewöhnlichen Krankheiten zu grunde gegangenen Personen (25), einbegriffen die chronischen und zu Erschöpfung führenden Erkrankungen (akute und chronische Affektionen des Respirationsapparates, auch tuberkulöse Formen, Infektionskrankheiten, kardio-vaskuläre Krankheiten, chronische Enteritiden, Marasma etc.).

Das Resultat war folgendes:

Volumen und Gewicht der Hoden bei den verschiedenen Formen von Geisteskrankheiten sind im größten Teil der Fälle - wobei das Alter der Individuen berücksichtigt ist mehr oder weniger unterhalb des Mediums, das als normal angesehen werden muß und das aus den Kontrollbeobachtungen erhalten wurde.

Diese Verminderung ist am auffälligsten bei den Formen von Paralyse, Dementia praecox, Psychosis alcoholica, P. epileptica, P. senilis und besonders bei Idiotie und Imbezillität, wo im allgemeinen die Geschlechtsdrüse enorm an Volumen reduziert ist. $\quad \mathrm{J}$. Ullmann (Rom).

Pardi, V. Untersuchungen über die spermatogenetische Funktion bei mit Kaffee vergifteten Tieren. La Sperimentale. Jahrg. LXV. Heft 1.

Pardis Resultate waren negativ. Die prolongierte Ingestion eines Kaffeeinfuses ist nicht ausreichend, die Testikularfunktion des Hundes zu beeinflussen, weder auf direktem Wege 
auf das Samenepithel, weder auf indirektem Wege vermittels des Zentralnervensystems. Der Kaffee scheint deshalb unschädlich für die Sexualfunktion zu sein, wenigstens bei den Hunden, die A. für seine Experimente benutzt hat.

J. Ullmann (Rom).

Bauereisen, A. Über die postoperative Infektion der weiblichen Harnorgane. Med. Kl. Nr. 22.

Nachdem B. anfangs seiner Arbeit darauf hinweist, wie lejcht die Blase und die Ureteren bei gynäkologischen und geburtshilflichen Operationen verietzt werden können, bespricht er die Blasenstörungen, die sich an gynäkologische Operationen anschließen können, ohne daß eine eigentliche Verletzung stattgefunden hat. Die meisten Schädigungen dieser Art kommen durch den Katheterismus zu stande, denn selbst bei peinlichster Asepsis läßt es sich nicht vermeiden, daß durch den Katheter Bakterien aus der Harnröhre in die Blase gebracht werden. Ist nun die Blase durch die Operation gleichsam wie durch ein Trauma geschädigt, so kommt es zu einer Zystitis, deren Erreger meistens Staphylokokken und Streptokokken sind, während der Koli-Bazillus allein fast nie eine Zystitis verursachen soll, sondern nur immer im Verein mit anderen Mikroorganismen. Die Symptome dieser Zystitiden sind dieselben wie bei jeder anderen akuten Blasenentzündung. Besonders weist der Autor auf die hämorrhagischen Zystitiden hin, die oft nur durch einen mikroskopischen Nachweis diagnostiziert werden können. Von der Blase her können natürlich durch die Ureteren die Nieren affiziert werden. Es kann dann zu Pyelitiden nnd eitrigen Nephritiden kommen. Daß eine postoperative Infektion der Harnnrgane das Resultat der Operation sehr beeinträchtigt, ist klar. Man soll daher mit dem Katheterismus sehr vorsichtig sein und eventuell versuchen, ohne ihn eine Blasenentleerung $\mathrm{zu}$ erreichen. Therapeutisch kommen dieselben Maßnahmen in Betracht, wie bei jeder anderen Zystitis.

Ludwig Z weig (Dortmund).

Baatz, Otto. Über Urethrektomie bei Harnröhrenstrikturen. Diss. Berlin 1911. Zusammenfassung aus Literaturangaben.

$$
\text { W eiler (Leipzig). }
$$

Backer, Max. Das Vulva-Karzinom in seiner Ätio$\log$ ie und Prognose. Diss. Erlangen 1912.

24 beobachtete Fälle. Fast ein Drittel der Fälle findet sich in dem Alter zwischen 66 und 70 Jahren, über zwei Drittel gehören dem fünften und sechsten Dezennium an. Die älteste Frau zählt über 79 Jahre, die jüngste 44 Jahre. Meist handelt es sich um Frauen in sehr gutem Ernährungszustand. 14 Fälle begannen als Oberflächenkrebs, 5 Erkrankungen 
müssen von vorneherein als infiltrierende Karzinome angesprochen werden. Ätiologisch $\mathrm{kam}$ meist die Einwirkung langdauernder Reizzustände in Frage. Die prognostisch ungüistigsten Primärtumoren sind Vulvourethralkarzinome und nächstdem die Klitoriskarzinome. Von den Drüsenmetastasen sind außer den infausten Beckentumoren prognostisch am ungünstigsten die karzinomatosen Ummauerungen der großen Schenkelgefäße. Die Prognose der nicht operierten Fälle war absolut tödlich. (Verlauf der Erkrankung zwischen $1^{1 / 2}$ und $2^{1 / 2}$ Jabren.) Von den Operierten war in 7 Fällen das Karzinom einigermaßen lokalisiert. Die Kranken blieben rezidivfrei. Alle übrigen Kranken starben mit einer Ausnahme. Als absolutes Heilungsr'esultat stellt sich also zirisa $28 \%$ heraus. Dieser ziemlich hohe Prozentsatz kann aber nicht als Dauerresultat bezeichnet werden, da nur 4 Fälle eine Rezidivfreiheit von zirka 5 Jahren haben. Danach bestünde ein Daverresultat von zirka $20 \%$.

$$
\text { Weiler (Leipzig). }
$$

Cecil, A. B. Eine Methode zur Ausführung der Urethrotomia externabei impermeabler Striktur. The Journal of the American Medical Association. 1913. Mai 24. pag. 1606.

Eingehende Darstellung der Technik, deren sich Cecil bei der Urothrotomia externa bedient mit Bericht über mehrere so operierte Fälle. Der Autor färbt durch vorhergehende Injektion von Methylenblau in die Urethra die Harnröhre und erleichtert sich so das Vorgehen.

Fritz Juliusberg (Posen).

Ayres, Winfield. Kolibazillus-Infektion der Niere. Medical Record. 1913. Mai 31. pag. 968.

A yres weist auf die Häufigkeit der hämatogenen Infektion der Niere durch den Bacillus coli hin. Prädisponierende Ursachen für diese Infektion der Niere sind Kongestion der Niere und präexistierende Entzündung. Unter die Kongestion gebören auch direkte und indirekte Traumen. Wir können hier auch die Fälle einreihen, welche sich an Gravidität, Operation an den Beckenorganen und Nierensteine anschließen. Die Diagnose muß kulturell geschehen, da der mikroskopische Nachweis nicht genügt. Behandeln soll man zunächst mit Hexamethylenamin und in der Dosis so weit gehen, bis man Formaldehyd im Urin nachweisen kann. (Nachweis nach Burnam: Zu der verdächtigen Flüssigkeit kommen 3 Tropfen einer $1 / 2 \%$ igen wässerigen Lösung von salzsauerem Phenylhydrazin, dann 3 Tropfen einer 5\% 5 igen wässerigen Lösung von Nitroprussidnatrium, dann ein Überschuß ron gesättigter wässeriger Natronlauge. Bei Vorhandensein von Formaldehyd auch nur in Konzentration von 1 zu 20.000 folgt eine intensiv 
blaue Verfärbung, welche nach und nach grün und nach einigen Minuten braun wird.) Wird kein Formaldehyd ausgeschieden, so wechsle man das Antiseptikum.

Fritz Julius berg (Posen).

Sinclair, J. D. S. Großes Fibromyom der Harnblase. The British Medical Journal. 1913. April 19. pag. 823.

Bericht über einen intravesikulären Tumor von Faustgröße, der gestielt in der Gegend des Trigonum bei einer 44 jährigen Frau aufsaß 3 und sich mikroskopisch als ein Fibromyom erwies.

Fritz Juliusberg (Posen).

Freyer, P. J. Eine Serie von 236 Fällen von totaler Enukleation der Prostata, ausgeführt während der zwei Jahre 1911-1912. The Lancet. 1913. April 12. pag. 1018.

Nicht zum kurzen Referat geeignet.

Fritz Juliusberg (Posen).

Coleman, C. A. Tuberkulin für die Behandlung der Urogenitaltuberkulose. Ohio State Medical Journal. 1913. Mai. Ref.: The Journal of the American Medical Association. 1913. Juni 14. pag. 1923.

Cole man kommt zu folgenden Schlüssen: Es gibt keine Spontanheilung bei Nierentuberkulose. Das Tuberkulin bringt keine Heilung, aber es wirkt unterstützend mit anderen Behandlungsmethoden kombiniert. Operable Fälle von Nierentuberkulose eignen sich nicht für Tuberkulininjektionen, aber frühe Fälle, bei denen sich Tuberkelbazillen ohne Eiterung oder Schädigung der funktionellen Aktivität der Niere finden, bis dann die Zeit zur Nephrektomie kommt. Die Tuberkulinbehandlung ist ferner indiziert, wenn nach der Nephrektomie noch Krankheitsherde im Harntraktus übrig bleiben.

Fritz Juliu sberg (Posen).

Spire, C. und Boeckel, André (Nancy). Geburtshilfliche Gründe für dieNephrektomiebei einseitiger Nierentuberkulose. Annales de Gynécologie et d'Obstetrique. 1913. Märzheft.

Das Vorhandensein nur einer Niere begründet keine Gefahr bei einer Schwangerschaft, während eine tuberkulöse Niere immer ein Weiterschreiten der Tuberkulose bei der Schwangerschaft befürchten läßt. Daher ist Verfasser für die Operation. Theodor S chwab (Karlsruhe).

Bass, Murray. Akute rheumatische Orchitis. Bericht über einen Fall mit gleichzeitigem Erythema nodosum und akuter Tortikollis bei einem $21 / 2 \mathrm{Jahr}$ alten Kinde. The Journal of the American Medical Association. 1913. Mai 24. pag. 1608.

Die Krankheit bei dem $2 \frac{1}{2}$ jährigen Kinde, über das 
Bass berichtet, begann mit Fieber, Üvelbefinden, Appetitlosigkeit und Erbrechen. Der Kopf war straff nach der linken Schulter geneigt. Papulöses Ekzem der Gesäßgegend, Erythema nodosum am linken Unterschenkel. Dazu trat eine Vergrößerung des linken Hodens. Heilung unter Salizylbehandlung. Ausführliche Mitteilung der diesbezüglichen Literatur.

Fritz Juliusberg (Posen).

Federici, N. Über die Indikationen der Orchiektomie bei der tuberkulösen Orchitis und Epididymitis. Gazz. d. Osp. e d. Clin. Nr. 7. 1912.

Nach Fe d erici rechtfertigt die tuberkulöse Orchiepididymitis käsiger oder käsig-eitriger Form, die mit Schmerzen oder Fieber einhergeht und den gewöhnlichen therapeutischen Mitteln trotzt, den chirurgischen Eingriff auch bei schon einseitig kastrierten Individuen, und selbst wenn schon andere, auch abdominale Lokalisationen existieren, vorausgesetzt, daß noch nicht das Terminalstadium gekommen ist.

J. Ullmann (Rom).

Stern, Heinrich. Die klinische Feststellung der Lymphurie. Medical Record. 1913. Mai 31. pag. 697.

Stern stellt in dieser Arbeit fest, erstens dab die Serumalbuminurie immer mit einer Globinurie verbunden ist, zweitens daß sich bei Lymphurie keine oder nur eine unbedeutende Globinurie findet, drittens daß Lymphurie ohne Zeichen einer Nierenschädigung vorkommt, viertens daß eine intermittierende juvenile Albuminurie ohne nachweisbare entzündliche Produkte, aber mit mehr oder weniger reichlichen Lymphozyten auf das Vorhandensein von Lymphe im Urin hinweist. Wenn bei solchem chemisch-mikroskopischen Befunde der Patient an einer intestinalen Erkrankung oder babituellen Obstipation leidet, wenn er Zeichen von Lymphstase. erweiterten Tonsillen, viel adenoidem Gewebe und palpablen Lymphdrüsen aufweist, wenn er anämisch ist, leicht erschöpft und körperlicher Arbeit abgeneigt, so ist die Diagnose einer Lymphurie gerechtfertigt.

Fritz Juliusberg (Posen).

Spitry, Hans. Ein Instrument zur radikalen Phimosenbeseitigung. Münch. med. Wochenschr. 1913. Nr. 18. Im Original nachzulesen.

Theodor B a er (Frankfurt a. M.).

Lißmann. Zur Behandlung der Pollutionen. Med. Kl. Nr. 22.

Nach LiBmann versteht man unter einer Pollution nur diejenigen Samenergüsse, die unwillkürlich mit Orgasmus und Wollustgefühl ruckweise vor sich gehen. Es sind daher die Prostatorrhöe, die Uretbrorrhöe ex libidine etc. auszuschließen. Dariiber, wann eine Pollution als normal zu gelten hat oder 
wann sie als pathologisch anzusehen ist, sind die Meinungen sehr geteilt. Jedenfalls läßt sich wohl sagen, daß, wenn eine Pollution als angenehm empfunden wird und weder geistige noch körperliche Sensationen hinterläßt, man sie als physiologisch ansehen kann. Hat sie, dagegen psychische Depressionen oder Schmerzen im Hinterkopf, Rücken oder Beinen etc. im Gefolge, so liegen zweifellos pathologische Verhältnisse vor. Als ätiologisches Moment krankbafter Pollutionen kommen alle chronischen Reize oder Entzündungen der Genitalorgane in Betracht; ebenfalls gehören hierher die Reize, die vom Gehirn ausgehen durch pornographische Lektüre etc. Ferner übt der Füllungszustand der Blase nicht selten einen starken, zur Pollution treibenden Reiz aus. Die. Therapie hat vor allem auf die $\ddot{A}$ tiölogie Rücksicht zu nehmen und muß je nach dem eingerichtet werden.

Zum Schluß empfiehlt L. ein Instrument zur Verhinderung der Pollution, "Antipolut" genannt. Es ist dies aus einem Federring hergestellter Apparat, der bei Eintritt einer Errektion einen Kontakt auslöst, wodurch eine elektrische Schelle eingeschaltet wird. Dadurch soll der Patient rechtzeitig gewarnt werden. Ludwig $\mathrm{Z}$ weig (Dortmund).

Underhill, Albert. Über sexuelle Neurasthenie beim Manne. The Journal of the American Medical Association. 1913. Juni 14. pag. 1869.

Underhill kommt in seiner Arbeit, die eine Anzahl von Krankengeschichten enthält, zu folgenden Schlüssen: 1. Die sexuelle Neurasthenie kommt immer durch definitive pathologische Zustände des Urogenitaltraktus zu stande. 2. Die Veränderungen können in verschiedenen Teilen des Traktus sitzen. 3. Da sich ganz besonders reichliche sensible Nervenendigungen in der prostatischen Urethra finden, so geben Veränderungen in ihr, besonders solche in der Nachbarschaft des Verumontanum, oft Anlaß zu diesem Typus der Neurasthenie. 4. Die Zustände geben die primäre Veranlassung zu einer lokalen Neuritis oder Neuralgie, welche die Zwischenursache für die Neurasthenie ist. 5. Es kommt manchmal ein toxisches Element vor, welches die Resistenz des sympathischen Nervensystems herabsetzt. Besonders häufig ist dies Vorkommen bei Infektionen der Samenblasen und der Prostata. 6. Der Zustand findet sich häufig bei Leuten mit hereditärer oder akquirierter neuropathischer Disposition. 7. Die physikalische Impotenz, zu der es bei diesen Fällen kommt, erhält im Laufe der Erkrankung eine psychische Komponente, welche besonders betrachtet werden muß, nachdem die primäre Ursache beseitigt ist.

Fritz Juliusberg (Posen). 
LiBmann. Ein Fall von seltener Potenzstörung. Dtsch. med. Woch. Nr. 21. 1913.

Der kräftige, nicht nervöse Patient Lißmanns hatte zwar nach langer Abstinenz in der Hochzeitsnacht eine Impotentia coeundi empfunden, nach wenigen Tagen aber normale Erektionsfähigkeit gezeigt. Dagegen trat 8 Jahre lang beim Koitus keine Ejakulation ein, welche auch durch Masturbation nur sehr selten zustande kam. Psychische Gründe erscheinen bei den einander sehr zugeneigten, Kindersegen wünschenden Eheleuten ausgeschlossen, hereditäre Belastung ebenso. $\mathrm{Ob}$ eine angeborene Anomalie oder die anerzogene Herabsetzung der Erregungsfähigkeit durch lange Enthaltsamkeit vorliegt, bleibt dahingestellt. Yohimbin sowie verschiedene Ratschläge blieben erfolglos. Max Jos eph (Berlin).

\section{Hautkrankheiten.}

\section{Allgemeines, Anatomie, Physiologie, allgem. und exper. Pathologie, path. Anatomie.}

Brugnatelli. Lipoidhaltige Zellen und Mastzellen in der Mamma. Soc. Med.-Chir. Pavia. Febr. 1913.

Brugnatelli hat im interstitiellen Gewebe der Mamma schwangerer Frauen Zellelemente von verschiedener Größe, mit reichlichem, granulösem, in der Quantität und Verteilung wechselndem Protoplasma gefunden, von denen einige Lipoide enthalten, andere lipoide Körnelungen und Körnelungen von Mastzellen.

In dieser vorläufigen Mitteilung geht A. nicht auf die Diskussion der Bedeutung dieses Befundes ein; er deutet nur die Hypothese an, daß Mastzellen, lipoidhaltige und interstitielle Zellen Elemente analogen Ursprungs sein können, die fähig sind, sich je nach den einwirkenden Reizen und nach den verschiedenen Organen zu transformieren.

$$
\text { J. Ullmann (Rom). }
$$

Unna, P. G. Zur Chemie der Zelle. Berl. klin. Woch. 1913. Nr. 18 und 19.

Studien der Chemie der Zelleiweiße auf dem Wege der Färbung, insbesondere des Granoplasmas und der Kernkörperchen. Max Leibkind (Dresden).

Krompecher. Vergleichende biologis ch-morphologische Studien betreffend die Fibroblasten und 